\section{COMPLEXITY OF FRESH HEVEA LATEX}

\author{
By Miss L. N. S. HOMANS, J. W. van DALFSEN \\ AND
}

G. E. van GILS

Netherlands Indies Rubber Research Institute, Buitenzorg, Java

$\mathrm{A}^{2}$ LTHOUGH under difficult conditions, the work at the Netherlands Indies Rubber Research Institute at Buitenzorg (Java) was continued during the Japanese occupation. Since it will be a long time before the accumulated results of the years 1942-45 can be published in detail, some points of general interest are reported here.

It is common knowledge that upon addition of small amounts of salts of bi- or poly-valent cations to fresh latex, a partial coagulation follows. The yellow-coloured coagulum generally contains 1030 per cent of the rubber and gives after drying a yellow rubber, rich in proteins and having a high acetone extract. The high acetone extract and the colour are produced by the yellow particles, first observed by Frey-Wyssling, which although generally called 'resin particles', contain mainly lipoids. What, however, is the reason of the high protein content?

In 1941 it was observed by one of us (L. N. S. H.) that when fresh latex is centrifuged in tubes, a separation occurs into a heavy yellow fraction, taking up a tenth to a third of the total volume, and a lighter white fraction. The yellow fraction is more viscous and often in itself consists of various layers, bright yellow, grey yellow, orange or even green coloured. Much information was obtained by studying separately the chemical composition, colloidchemical and enzymological properties of these fractions.

The difference in composition is evident from the following example:

\begin{tabular}{|c|c|c|c|}
\hline & $\underset{\text { latex }}{\text { Original }}$ & $\begin{array}{l}\text { White } \\
\text { fraction }\end{array}$ & $\begin{array}{l}\text { Yellow } \\
\text { fractior }\end{array}$ \\
\hline $\begin{array}{l}\text { Ratio of weight } \\
\text { Specific gravity } \\
\text { Viscosity (centipoises) } \\
\text { Dry rubber content }(\%) \\
\text { Total solids } \\
\text { Nitrogen content } \\
\text { Ash content } \\
\begin{array}{ll}\text { pH (fresh) } & (\%) \\
\text { ( })\end{array} \\
\end{array}$ & $\begin{array}{l}100 \\
0 \cdot 977 \\
11 \cdot 7 \\
34 \cdot 8 \\
37 \cdot 4 \\
0 \cdot 24 \\
0 \cdot 60 \\
6 \cdot 3\end{array}$ & $\begin{array}{l}76 \cdot 3 \\
0 \cdot 966 \\
9 \cdot 2 \\
43 \cdot 7 \\
45 \cdot 8 \\
0 \cdot 22 \\
0 \cdot 47 \\
6 \cdot 9\end{array}$ & $\begin{array}{c}23 \cdot 7 \\
? \\
? \\
9 \cdot 0 \\
14 \cdot 3 \\
0 \cdot 33 \\
1 \cdot 11 \\
5 \cdot 7\end{array}$ \\
\hline \multicolumn{4}{|c|}{ Crepes prepared from the sample had: } \\
\hline $\begin{array}{ll}\text { Nitrogen content }(\%) \\
\text { Ash content } & (\%) \\
\text { Acetone extract } & (\%)\end{array}$ & $\begin{array}{l}0 \cdot 37 \\
0 \cdot 15 \\
2 \cdot 42\end{array}$ & $\begin{array}{l}0 \cdot 34 \\
0 \cdot 13 \\
2 \cdot 10\end{array}$ & $\begin{array}{l}0 \cdot 73 \\
0 \cdot 50 \\
6 \cdot 99\end{array}$ \\
\hline
\end{tabular}

It is clear that a large part of the non-rubber constituents of the latex assembles in the yellow fraction, whereas the white fraction is a purer rubber dispersion than the original latex. In spite of its higher rubber content, the white fraction is less viscous, even after ammoniation, and consequently it has better creaming properties.

Another important difference is in their behaviour on standing. Whereas the original latex coagulates spontaneously after about twelve hours, the white fraction may stay fluid for days. The yellow fraction, on the other hand, coagulates within a few hours. This probably is a consequence of the fact that all enzymatic reactions in the yellow fraction are much more pronounced than in the white fraction, giving rise to accelerated splitting of large-molecule complexes and the formation of free fatty acids which, in their turn, in the presence of magnesium ions, effect spontaneous coagulation ${ }^{1}$. The addition of a freshly made pancreas lipase extract accelerates the spontaneous coagulation of the yellow fraction considerably, is less active in the original latex and has scarcely any influence on the white fraction.

A clear demonstration of the greater activity of enzymes in the yellow fraction is given by the dark discoloration on the surface, which is caused by oxidizing enzymes. On standing exposed to air, latex sometimes becomes discoloured on the surface, turning grey or greyish-purple. The white fraction never becomes discoloured; but the yellow fraction always becomes dark grey or purplish-black, often very quickly. Gas formation occurs in the yellow fraction earlier and to a much greater extent than in the white fraction.

Microscopic observations. The complexity of fresh latex, caused by the existence of the yellow fraction, is even observable under the microscope provided the latex is examined in a thin layer and with a small magnification (60-200) and that it is not diluted. The rubber dispersion then is visible as a highly absorbent mass ; the rubber particles are not visible separately; but the whole mass is constantly glittering and twinkling in consequence of Brownian movement. The yellow fraction, on the other hand, appears as irregular shaped transparent 'islands' with an irregular structure. The great difference in viscosity is evident. By pressing on the cover glass, streaming of the rubber dispersion is easily effected; but the bright 'islands' are viscous and change their shape only slowly and with difficulty. The phenomenon is especially clearly observable in latex from twigs and from young trees, since in such lattices the yellow fraction takes up a large, even the larger, part of the volume.

Stability. In old ammoniated latex the yellow fraction is no longer present. Resin particles may be observed, but not the viscous matter just described. This is easily understood by observing under the microscope the picture which appears when alkali diffuses into fresh latex: the transparent viscous islands dissolve. Sometimes, however, the -viscous matter is more resistant and in exceptional cases it was still present in ammoniated latex several weeks old. Not only the amount but also the properties are dependent on all kinds of biological factors, such as age and strain of the tree, place and frequency of tapping; possibly the season, the weather, even the soil may be of influence.

Very much in contrast to the influence of alkali is the action of distilled water. Now the viscous matter may be observed to stiffen and flocculate under the microscope. From this observation puzzling effects such as the rise in viscosity upon dilution of fresh latex with water, and the forming of clots upon dialysing, now become clear. The behaviour of the yellow fraction of Hevea latex upon addition of water is analogous to that of Cryptostegia latex ${ }^{2}$, since coagulation results.

Dilution of the yellow fraction without a change in the consistency of the viscous matter is possible with a salt solution containing a monovalent cation of about 0.1 normality. With such a solution it becomes possible to wash the yellow fraction by repeated centrifuging, decanting and diluting. Moreover, under suitable conditions as to $p \mathrm{H}$ and the concentration of the monovalent cation, it is possible to separate the yellow fraction without centrifuging 
but simply by gravity. The fact that the addition of salt reduces the viscosity of the fresh latex very considerably is one of the reasons for this.

The true nature of the viscous matter in fresh latex is not yet known, but the properties resemble those of coacervates ${ }^{3}$ very much; thus it seems probable that fresh latex contains a micro-coacervate which by centrifuging or under favourable conditions as to the viscosity goes over in a macro-coacervate.

The progress of our research work has been slowed down, first by the War and later it was interrupted by the unhappy conditions in Java; it is expected, however, that work at our Institute will be resumed in the near future.

${ }^{1}$ Compare v. Gils, Arch. Rubbercultuur, 25, 395 (1941).

Symontowne, Ind. Rubb. W.108.148259 (1943).

' See Kruyt and Bungenberg de Jong, Koll. Z.. 50, 39 (1930).

\section{STUDIES IN STOMATAL ACTION}

\section{Adequacy of the Porometer in the Investigation of Stomatal Aperture}

$I^{\mathrm{N}}$ the usual form of porometer a small cup is permanently attached to the leaf surface, air is drawn through the leaf into the cup under reduced pressure and either the rate of air flow is measured ${ }^{1,2}$ or, in the 'resistance porometer' 3,4 , the fall in pressure across the leaf and also that across a standard capillary resistance in series is found; from these pressure gradients the resistance to flow through the leaf is calculated. The rate of flow or the resistance to flow are functions, though very complex ones, of stomatal aperture. In earlier work porometers were used for the purely qualitative purpose of showing whether the stomata were opening or closing. Interest in the dynamics of stomatal movement $t^{5,6}$ and in the relation between stomatal movement and carbon assimilation ${ }^{2,8}$, however, made it desirable to attempt to estimate from porometer readings the resistance to viscous flow of the stomata alone, thus eliminating the confusing effects of the irrelevant internal resistances in the leaf. Hence, ultimately, making use of the less convenient 'diffusion porometer' ${ }^{\prime}$ to calibrate the 'resistance porometer', the resistance to diffusion through the stomata might be estimated. With this end in view a mathematical theory of porometers was elaborated ${ }^{10,4}$ and used to estimate stomatal resistances in Pelargonium 4 .

During a recent investigation of shock-induced stomatal movements ${ }^{11}$, it was found that with stomata open in the light, strips of epidermis taken by Lloyd's ${ }^{12}$ method within the cup area showed very much wider apertures than those taken just outside the cup. This was at first attributed to the lack of shock sensitivity of the stomata within the cup ${ }^{11}$. The possibility existed, however, that the stomata outside the cup were not showing 'shock closure' on stripping of the epidermis, but actually had smaller apertures on the intact leaf than those within the cup. This, if true, would have an important bearing on the quantitative use of porometers, for the theory mentioned above demands the assumption that the stomatal apertures are the same inside and outside the cup.

A considerable number of experiments, carried out separately after consultation between us, has left no doubt that illuminated leaves, both of Pelargonium zonale and Triticum vulgare, have, in fact, much wider stomatal apertures where the surface is enclosed in a cup than elsewhere. This has been shown by a modification of Molisch's ${ }^{13}$ injection technique using gentian violet in absolute alcohol (Pelargonium and Triticum) as well as by Lloyd's method and direct microscopical observation (Pelargonium). If part of the lamina of a Pelargonium or wheat leaf is merely placed between two glass strips and then, after two or three hours illumination, flooded with alcohol, heavy injection indicating wide open stomata is found where the leaf has been enclosed, and little or none elsewhere. Darkened portions show no injection either within or outside the enclosed area. Similar results are obtained using small chambers resembling porometer cups.

The conditions within a porometer cup differ from those outside in the following respects: (1) The air inside the cup is of restricted volume and at rest between readings (if the porometer is disconnected); it thus rapidly becomes saturated with water vapour, a process which is hastened by the drawing of air from the intercellular space system of the leaf into the cup during readings. In the case of Pelargonium, the concentration of ethereal oils must also be higher within the cup; with any species other gaseous substances may diffuse out of the leaf, and the oxygen and carbon dioxide tensions in the cup will also be affected. (2) The upper surface of the leaf above the cup is (normally) touching a glass plate. (3) The temperature within the cup may be slightly higher. (4) The leaf in the cup area is (usually) illuminated from above only, the cup shading the lower surface from any direct light.

The possible effects of these differences may now be considered.

(1) Experiments, each witn a pair of porometer cups on the same leaf, one cup containing a desiccating agent (silica gel or calcium chloride) and the other empty or with moist broken porous tile, show similar wide stomatal opening in both cups, whether judged by porometer readings or Lloyd's method. These results seem to dispose of high humidity as the factor causing the wide opening within the cup. The ethereal oils of Pelargonium also seem unlikely to be important in this respect, for similar effects are found with wheat. The question of other variations in the composition of the air in the cup is discussed elsewhere ${ }^{14}$.

(2) It is found that similar wide stomatal opening occurs when Pelargonium or wheat plants are enclosed in a bell jar, in a saturated atmosphere, with the leaves not in contact with the glass. Thus pressure of the glass plate on the leaf is not concerned. Similar plants outside the bell jar show little opening except where the leaves are enclosed between glass plates, as above.

(3) In the bell jar experiments just cited the difference in temperature inside and outside does not exceed $1 \frac{1}{2}^{\circ} \mathrm{C}$. Thus the differences in aperture can arise in the absence of appreciable temperature differences.

(4) These experiments have been carried out with moderate light intensities-a 100-W. or 150-W. lamp, with running water screen, at 3-7 in. distance. It seems improbable that reducing the illumination of the lower surface of the leaf would cause wider stomatal opening. In all cases the usual closing response to darkening the upper surface has been found.

Whatever the cause of the wider opening within the cup, and this is the subject of a further com- 